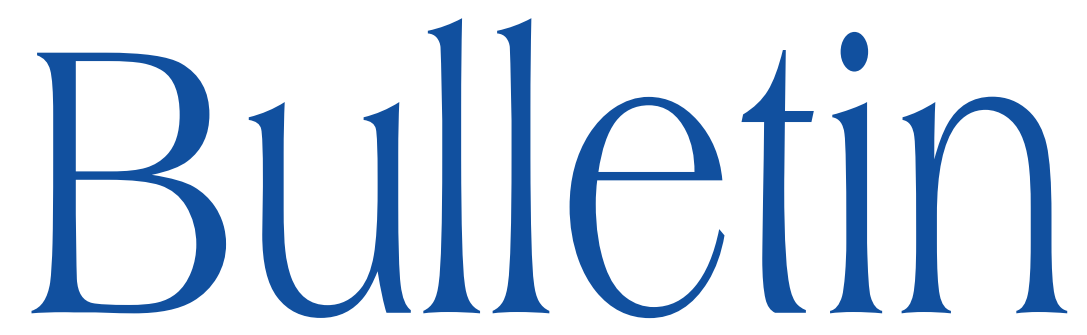

de la SOCIÉTÉ MATHÉMATIQUE DE FRANCE

\title{
HOMOTOPY INVARIANCE OF HIGHER SIGNATURES AND 3-MANIFOLD GROUPS
}

Michel Matthey \& Hervé Oyono-Oyono \& Wolfgang Pitsch

Tome 136 Fascicule 1

2008 
Bull. Soc. math. France

136 (1), 2008, p. 1-25

\title{
HOMOTOPY INVARIANCE OF HIGHER SIGNATURES AND 3-MANIFOLD GROUPS
}

\author{
By Michel Matthey, Hervé Oyono-Oyono \\ \& WOLFGANG Pitsch
}

To the memory of Michel Matthey

\begin{abstract}
For closed oriented manifolds, we establish oriented homotopy invariance of higher signatures that come from the fundamental group of a large class of orientable 3-manifolds, including the "piecewise geometric" ones in the sense of Thurston. In particular, this class, that will be carefully described, is the class of all orientable 3-manifolds if the Thurston Geometrization Conjecture is true. In fact, for this type of groups, we show that the Baum-Connes Conjecture With Coefficients holds. The non-oriented case is also discussed.
\end{abstract}

Texte reçu le 27 janvier 2006, révisé le 11 octobre 2006

Michel Matthey

Hervé Oyono-Oyono, Université Blaise Pascal à Clermont-Ferrand, Département de mathématiques, Complexe Scientifique des Cézeaux, 63177 Aubière Cedex (France)

E-mail : oyono@math.univ-bpclermont.fr

Wolfgang Pitsch, Universitat Autònoma de Barcelona, Departament de Matematiquès, E-08193 Bellaterra (Spain) • E-mail : pitsch@mat.uab.es

2000 Mathematics Subject Classification. - 19K35, 57R20, 57M50, 46L80.

Key words and phrases. - Baum-Connes conjecture, JSJ decomposition, Thurston geometrization conjecture.

The third author is supported by MEC Grant MTM2004-06686 and by the program Ramón y Cajal, MEC, Spain. 
RÉSUMÉ (Invariance homotopique des signatures supérieures et groupes fondamentaux des variétés de dimension 3)

Nous démontrons que pour des variétés fermées et orientées les signatures qui proviennent des groupes fondamentaux d'une large classe de variétés orientables de dimension 3 sont des invariants homotopiques. Cette classe, que nous décrivons soigneusement, contient en particulier les variétés géométriques par morceaux au sens de Thurston. Si la conjecture de géométrisation de Thurston s'avère vraie cette classe coïncide alors avec celle des groupes fondamentaux de variétés de dimension 3 orientables. Plus précisément nous démontrons que tous les groupes dans cette classe satisfont la conjecture de Baum-Connes avec coefficients. Nous discutons également le cas non-orientable.

\section{Introduction and statement of the main results}

We assume all manifolds to be non-empty, pointed (i.e. we fix a base-point), second countable, Hausdorff and smooth. Given a closed connected oriented manifold $M^{m}$ of dimension $m$, let $[M]$ denote either orientation classes in $H_{m}(M ; \mathbb{Q})$ and in $H^{m}(M ; \mathbb{Z})$, and let $\mathcal{L}_{M} \in H^{4 *}(M ; \mathbb{Q})$ be the Hirzebruch $L$-class of $M$, which is defined as a suitable rational polynomial in the Pontrjagin classes of $M$ (see [23, pp. 11-12] or [37, Ex. III.11.15]). Denote the usual Kronecker pairing for $M$, with rational coefficients, by

$$
\langle., .\rangle: H^{*}(M ; \mathbb{Q}) \times H_{*}(M ; \mathbb{Q}) \longrightarrow \mathbb{Q} .
$$

If $M$ is of dimension $m=4 k$, then the Hirzebruch Signature Theorem (see [23, Thm. 8.2.2] or [37, p. 233]) says that the rational number $\left\langle\mathcal{L}_{M},[M]\right\rangle$ is the signature of the cup product quadratic form

$$
H^{2 k}(M ; \mathbb{Z}) \otimes H^{2 k}(M ; \mathbb{Z}) \longrightarrow H^{4 k}(M ; \mathbb{Z})=\mathbb{Z} \cdot[M] \cong \mathbb{Z}, \quad(x, y) \longmapsto x \cup y .
$$

As a consequence, $\left\langle\mathcal{L}_{M},[M]\right\rangle$ is an integer and an oriented homotopy invariant of $M$ (among closed connected oriented manifolds, hence of the same dimension $4 k$ ). In 1965, S.P. Novikov proposed the following conjecture, now known as the Novikov Conjecture or as the Novikov Higher Signature Conjecture: Let $G$ be a discrete group, let $B G$ be its classifying space, and let $\alpha \in H^{*}(B G ; \mathbb{Q}) \cong H^{*}(G ; \mathbb{Q})$ be a prescribed rational cohomology class of $B G$. Now, for a closed connected oriented manifold $M^{m}$ (with $m$ arbitrary) and for a continuous map $f: M \longrightarrow B G$, consider the $\alpha$-higher signature (coming from $G$ )

$$
\operatorname{sign}_{\alpha}^{G}(M, f):=\left\langle f^{*}(\alpha) \cup \mathcal{L}_{M},[M]\right\rangle \in \mathbb{Q},
$$

TOME $136-2008-\mathrm{N}^{\mathrm{O}} 1$ 
where $f^{*}: H^{*}(B G ; \mathbb{Q}) \longrightarrow H^{*}(M ; \mathbb{Q})$ is induced by $f$. Then, the conjecture predicts that the rational number $\operatorname{sign}_{\alpha}^{G}(M, f)$ is an oriented homotopy invariant of the pair $(M, f)$, in the precise sense that $\operatorname{sign}_{\alpha}^{G}(N, g)=\operatorname{sign}_{\alpha}^{G}(M, f)$ whenever $N^{n}$ is a second closed connected oriented manifold equipped with a continuous map $g: N \longrightarrow B G$, and such that there exists a homotopy equivalence $h: M \stackrel{\simeq}{\longrightarrow} N$ preserving the orientation, that is, $h_{*}[M]=[N]$ in $H_{m}(N ; \mathbb{Q})$ (automatically, $m=n$ ), and with $g \circ h \simeq f$, i.e. the diagram

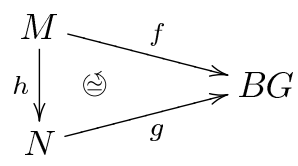

commutes up to homotopy, as indicated. If, for a given group $G$, this holds for every rational cohomology class $\alpha \in H^{*}(B G ; \mathbb{Q})$, then one says that $G$ verifies the Novikov Conjecture. Of particular interest are the "self higher signatures" of a closed connected oriented manifold $M$, namely those corresponding to the case $G:=\pi_{1}(M)$, for some chosen cohomology class $\alpha \in H^{*}(B G ; \mathbb{Q})$, with, as map $f: M \longrightarrow B G$, 'the' classifying map of the universal covering space $\widetilde{M}$ of $M$ (up to homotopy). Special attention is deserved by the situation where $M$ is aspherical, in which case one can take $M$ as a model for $B G$, and $f:=\operatorname{id}_{M}$.

Now, fix a discrete group $G$ (countable, say) and let $\mathbb{C} G$ be the complex group algebra of $G$. Then $\mathbb{C} G$ is equipped with an involution

$$
\lambda_{g_{1}} g_{1}+\cdots+\lambda_{g_{k}} g_{k} \longmapsto \bar{\lambda}_{g_{1}} g_{1}^{-1}+\cdots+\bar{\lambda}_{g_{k}} g_{k}^{-1}
$$

and any unitary representation $U$ of $G$ on a Hilbert space $H_{U}$ gives rise to an involutive representation $\pi_{U}$ of $\mathbb{C} G$ on $H_{U}$. The maximal $C^{*}$-algebra of $G$, denoted by $C^{*} G$ is then the completion of $\mathbb{C} G$ with respect to the norm

$$
\|\bullet\|_{\max }:=\sup _{U}\left\|\pi_{U}(\bullet)\right\|_{H_{U}},
$$

where $U$ runs through all unitary representations of $G$. On the other hand, the reduced $C^{*}$-algebra of $G$, denoted by $C_{r}^{*} G$, is by definition the completion of $\mathbb{C} G$ with respect to the norm

$$
\|\bullet\|_{r}:=\left\|\pi_{\lambda}(\bullet)\right\|_{\ell^{2}(G)},
$$

where $\lambda$ is the left regular representation, i.e the representation of $G$ on $\ell^{2}(G)$ given by left translations. Notice that we have an obvious surjective map

$$
\lambda^{G}: C^{*} G \longrightarrow C_{r}^{*} G .
$$

Let $K_{*}(-)$ and $K_{*}^{\text {top }}(-)$ denote respectively complex topological $K$ homology with compact supports for spaces and analytical $K$-theory for complex Banach algebras. In [42], Miščenko defines a group homomorphism

$$
\tilde{\nu}_{*}^{G}: K_{*}(B G) \longrightarrow K_{*}^{\text {top }}\left(C^{*} G\right)
$$


and shows that if $\tilde{\nu}_{*}^{G}$ is rationally injective, i.e. injective after tensoring with $\mathbb{Q}$, then the Novikov Conjecture holds for $G$. The composite

$$
\nu_{*}^{G}: K_{*}(B G) \stackrel{\tilde{\nu}_{*}^{G}}{\longrightarrow} K_{*}^{\mathrm{top}}\left(C^{*} G\right) \stackrel{\lambda_{*}^{G}}{\longrightarrow} K_{*}^{\mathrm{top}}\left(C_{r}^{*} G\right)
$$

is called the Novikov assembly map and the so-called Strong Novikov Conjecture for $G$ is the statement that $\nu_{*}^{G}$ is rationally injective, and this, again, implies the usual Novikov Conjecture. Next, we explain the connection with the BaumConnes Conjecture. Let $\underline{E G}$ denote the universal example for proper actions of $G$ (in other words, up to $G$-homotopy, the classifying space for the family of finite subgroups of $G$ ); by definition, this is a locally compact Hausdorff proper (left, say) $G$-space such that for any locally compact Hausdorff $G$-space $X$, there exists a $G$-map from $X$ to $\underline{E G}$, and any two $G$-maps from $X$ to $\underline{E G}$ are $G$-homotopic. For instance, the universal covering space $E G:=\widetilde{B G}$ of $B G$ is a model for $\underline{E G}$ when $G$ is torsion-free; the point $p t$ is a model for $\underline{E G}$ when $G$ is finite; if $G$ is a discrete subgroup of an almost connected Lie group $\Gamma$ with maximal compact subgroup $K$, then $\Gamma / K$ is a model for $\underline{E G}$. Suppose further given a separable $G-C^{*}$-algebra $\mathcal{A}$. Then, there is a suitable $G$-equivariant $K$ homology group $K_{*}^{G}(\underline{E G} ; \mathcal{A})$ and a specific group homomorphism, called the Baum-Connes assembly map with coefficients in $\mathcal{A}$,

$$
\mu_{*}^{G, \mathcal{A}}: K_{*}^{G}(\underline{E G} ; \mathcal{A}) \longrightarrow K_{*}^{\mathrm{top}}\left(\mathcal{A} \rtimes_{r} G\right),
$$

where $\mathcal{A} \rtimes_{r} G$ is the reduced $C^{*}$-crossed product of $\mathcal{A}$ by $G$. The group $G$ is said to satisfy the Baum-Connes Conjecture With Coefficients if the assembly map $\mu_{*}^{G, \mathcal{A}}$ is an isomorphism for any separable $G-C^{*}$-algebra $\mathcal{A}$. If this is at least known to be fulfilled for the $C^{*}$-algebra $\mathbb{C}$ with trivial $G$-action, then one says that $G$ verifies the Baum-Connes Conjecture (i.e. without mentioning coefficients). In this special case where $\mathcal{A}=\mathbb{C}$ with trivial $G$-action, one has $\mathcal{A} \rtimes_{r} G=C_{r}^{*} G$ and $K_{*}^{G}(\underline{E G} ; \mathcal{A})=K_{*}^{G}(\underline{E G})$, the $G$-equivariant $K$-homology group with $G$-compact supports of $\underline{E G}$, and the corresponding assembly map boils down to a map

$$
\mu_{*}^{G}:=\mu_{*}^{G, \mathbb{C}}: K_{*}^{G}(\underline{E G}) \longrightarrow K_{*}^{\mathrm{top}}\left(C_{r}^{*} G\right) .
$$

This is linked with the Novikov Conjecture as follows. First, since $G$ acts properly and freely on $E G$, and since $B G \simeq G \backslash E G$, there is a canonical isomorphism

$$
K_{*}(B G) \cong K_{*}^{G}(E G) .
$$

Secondly, since tautologically any proper and free $G$-action is proper, there is a $G$-map $E G \longrightarrow \underline{E G}$, unique up to $G$-homotopy, and the induced map

$$
K_{*}^{G}(E G) \longrightarrow K_{*}^{G}(\underline{E G})
$$


is known to be rationally injective. Thirdly, the Novikov assembly map $\nu_{*}^{G}$ coincides with the composite map

$$
K_{*}(B G) \cong K_{*}^{G}(E G) \longrightarrow K_{*}^{G}(\underline{E G}) \stackrel{\mu_{*}^{G}}{\longrightarrow} K_{*}^{\mathrm{top}}\left(C_{r}^{*} G\right) .
$$

It follows that if the group $G$ satisfies the Baum-Connes Conjecture (in particular, if $G$ verifies the Baum-Connes Conjecture With Coefficients), then the Strong Novikov Conjecture holds for $G$, and hence also the original Novikov Conjecture on higher signatures. If the group $G$ is torsion free, then as we mentionned before, we can choose $E G$ as a model for the universal example for proper action and thus, up to the identification between $K_{*}(B G)$ and $K_{*}^{G}(E G)$, the Baum-Connes and the Novikov assembly maps coincides. At this point, we shall take the opportunity to explain why these assembly maps have to be valued in the reduced $C^{*}$-algebra of $G$ rather than in the maximal one if we expect surjectivity for the assembly map.

The trivial representation of $G$ induces a morphism $\pi: C^{*} G \rightarrow \mathbb{C}$ and if we denote by $e$ the neutral element of $G$, the map

$$
\mathbb{C} G \ni \lambda_{g_{1}} g_{1}+\cdots+\lambda_{g_{k}} g_{k} \longmapsto \lambda_{e}
$$

extends to a trace $\operatorname{tr}: C^{*} G \rightarrow \mathbb{C}$ factorizing through $C_{r}^{*} G$. According to Atiyah's Index Theorem for coverings [3] on one side and to the naturality of the assembly map [41] on the other side, the morphisms induced on $K$-theory by $\pi$ and $\operatorname{tr}$ should coincide on the range of Miščenko morphism $\tilde{\nu}_{*}^{G}: K_{*}(B G) \longrightarrow K_{*}^{\text {top }}\left(C^{*} G\right)$. In consequence, if $G$ is torsion free and has Kazhdan property $(T)$, then the class in $K$-theory of the projector $p$ of $C^{*} G$ corresponding to the projection on invariants can not be in the range of Miščenko morphism, since $\pi(p)=1$ and $\operatorname{tr}(p)=0$. In contrast, we shall see in Section 3.1 that, up to Thurston hyperbolization conjecture, the natural map

$$
K_{*}^{\mathrm{top}}\left(C^{*} G\right) \stackrel{\lambda_{*}^{G}}{\longrightarrow} K_{*}^{\mathrm{top}}\left(C_{r}^{*} G\right)
$$

is an isomorphism when $G$ is the fundamental group of a compact orientable 3-manifold. As general references for the Baum-Connes Conjecture and related topics, let us mention [5], [6], [41], [52].

In this paper, we observe that so much is known about the structure of 3manifolds and that the Baum-Connes Conjecture With Coefficients has been proved for such a large class of groups, that this enables to establish the BaumConnes Conjecture With Coefficients for the fundamental group of any compact orientable 3-manifold "with a piecewise geometric structure", more precisely to which the famous Thurston Geometrization Conjecture applies, namely:

THEOREM 1.1. - Suppose that the Thurston Hyperbolization Conjecture is true, as for example if the Thurston Geometrization Conjecture holds. Let $G$ 
be the fundamental group of an orientable 3-manifold, compact or not, with or without boundary. Then, the Baum-Connes Conjecture With Coefficients holds for $G$. In particular, the group $G$ satisfies the Novikov Conjecture, i.e. higher signatures coming from $G$ are oriented homotopy invariants for closed connected oriented manifolds of arbitrary dimension.

Remark 1.2. - In Section 2, more details will be given about the Thurston Geometrization Conjecture and the Thurston Hyperbolization Conjecture (see Remark 2.1 below).

Remark 1.3. - By recent outstanding results of Perelman, one might expect to have, in a near future, a complete proof of the Thurston Geometrization Conjecture, and hence of the Thurston Hyperbolization Conjecture.

In fact, in the compact case, we have a more precise result, independently of the Thurston Hyperbolization Conjecture:

THEOREM 1.4. - Let $G$ be the fundamental group of a compact orientable 3manifold $M$ (possibly with boundary), and consider a two-stage decomposition of the capped-off manifold $\widehat{M}$ of $M$, firstly, into Kneser's prime decomposition, secondly, for each occurring closed irreducible piece with infinite fundamental group, a Jaco-Shalen-Johannson torus decomposition. Now, consider only those pieces obtained after the second stage and which are closed, non-Haken, non-Seifert, non-hyperbolizable and whose fundamental group is infinite. Suppose that the fundamental groups of these very pieces satisfy the Baum-Connes Conjecture with Coefficients. Then, $G$ verifies the Baum-Connes Conjecture with Coefficients and the Novikov Conjecture.

REmark 1.5. - Let $M$ be a compact 3-manifold. The capped-off manifold $\widehat{M}$ of $M$ is obtained from $M$ by capping off with a compact 3-ball each boundary component of $M$ that is diffeomorphic to a 2 -sphere, getting this way a compact 3-manifold $\widehat{M}$, see [19, p. 25]. Note that $\widehat{M}$ is orientable whenever $M$ is orientable, and that the inclusion $M \hookrightarrow \widehat{M}$ induces an isomorphism on fundamental groups.

Remark 1.6. - In Section 2, we will explain Kneser's and Jaco-ShalenJohannson's decompositions. We will also recall the notions of prime, of irreducible, of Haken, of Seifert, and of hyperbolizable 3-manifolds. In particular we will see that the "exotic pieces" in Theorem 1.4 only appear when no further decomposition is possible after Kneser's. 
REMARK 1.7. - In particular, all "self higher signatures" are oriented homotopy invariants for closed connected oriented 3-manifolds to which Theorems 1.1 and 1.4 apply. At this point, it is worth mentioning that all irreducible compact connected orientable 3-manifolds with infinite fundamental group are aspherical, as follows from the Sphere Theorem, see [48, p. 483] and [19, Thm. 4.3].

In the non-orientable compact case, we have the following result.

THEOREM 1.8. - Let $M$ be a compact non-orientable 3-manifold, and let $G$ be its fundamental group. Let $M_{1}, \ldots, M_{p}$ be the irreducible pieces in Kneser's (normal) prime decomposition. Suppose, for each $i=1, \ldots, p$, that one of the following properties is fulfilled: either $M_{i}$ is orientable and satisfies the hypotheses of Theorem 1.4 (as for example if the Thurston Hyperbolization Conjecture is true); or $\pi_{1}\left(M_{i}\right)$ is infinite cyclic; or $M_{i}$ is non-orientable and without 2-torsion in its fundamental group. Then, the group $G$ satisfies the Baum-Connes Conjecture With Coefficients and the Novikov Conjecture.

REMARK 1.9. - In Section 2, we will explain when a Kneser prime decomposition is called normal (a property guaranteeing its uniqueness).

Remark 1.10. - The Baum-Connes Conjecture With Coefficients, hence the Novikov Conjecture, is known for the fundamental group of any manifold of dimension $\leq 2$. So, what is done here, is extending this result up to dimension 3 in the orientable case, modulo the Thurston Hyperbolization Conjecture. Since, for each $n \geq 4$, every finitely presentable group is isomorphic to the fundamental group of some closed connected orientable (smooth) $n$-manifold (see for instance [13], [38] or [28]), a further extension one dimension up should certainly be incomparably more difficult and seems to be, by far, out of scope at the time of writing. At this point, we mention that by an unpublished result of Connes, Gromov and Moscovici (see however [18]), for closed connected oriented manifolds of arbitrary dimension, all higher signatures coming from a discrete group $G$ and corresponding to a cohomology class lying in the subring of $H^{*}(B G ; \mathbb{Q})$ generated by the classes of degree $\leq 2$ are oriented homotopy invariants; a complete proof is now available in [39, Cor. 0.3].

Remark 1.11. - In Theorems 1.1, 1.4 and 1.8, one does not need to suppose that the considered 3-manifolds are smooth manifolds, but merely topological manifolds. Indeed, as is well-known, any (second countable Hausdorff) topological manifold of dimension $\leq 3$ admits a smooth structure, which is furthermore unique. 
REMARK 1.12. - If it would be known that any countable discrete group $G$ sitting in a short exact sequence of groups

$$
1 \rightarrow H \longrightarrow G \longrightarrow \mathbb{Z} / 2 \rightarrow 1,
$$

with $H$ satisfying the Baum-Connes Conjecture With Coefficients, verifies itself the Baum-Connes Conjecture With Coefficients, then one could drop the condition "orientable" in Theorems 1.1 and 1.4 (one could also drop the first occurring assumption of orientability in Theorem 1.13 below). Indeed, there is no restriction in assuming connectedness of the considered 3-manifold $M$ (which is compact for 1.4), and in case $M$ is non-orientable, Theorems 1.1 or 1.4 hold for the orientation covering $\bar{M}$ of $M$, which is a regular double covering of $M$ (and is itself compact for 1.4). One has the fibre sequence $S^{0} \rightarrow \bar{M} \rightarrow M$ and therefore a short exact sequence of groups

$$
1 \rightarrow \pi_{1}(\bar{M}) \longrightarrow \pi_{1}(M) \longrightarrow \mathbb{Z} / 2 \rightarrow 1 \text {. }
$$

Recall that for a torsion-free discrete group $G$, the Kaplansky/Idempotent Conjecture (resp. the Kadison-Kaplansky Conjecture) states that the algebra $\mathbb{C} G$ (resp. $C_{r}^{*} G$ ) contains no non-trivial idempotent, i.e. any element $\varepsilon$ satisfying $\varepsilon=\varepsilon^{2}$ is equal to 0 or 1 .

Theorem 1.13. - Suppose that the Thurston Hyperbolization Conjecture is true. Then, Kaplansky's Idempotent Conjecture and the Kadison-Kaplansky Conjecture hold for any torsion-free fundamental group of an orientable 3manifold, as for example for the fundamental group of any compact orientable 3-manifold whose prime factors in Kneser's prime decomposition all have an infinite fundamental group.

REMARK 1.14. - Of course, there is a analogous statement to Theorem 1.13 for all fundamental groups to which Theorem 1.4 applies, provided they are torsion-free.

Notice to the reader. - Section 2 reflects the origin of this paper: $K$-theorists meeting low-dimensional topologists and learning the ones from the others their subjects. therefore we shall enter in some detail of the proofs in the hope that readers from one area will find it usefull as an introdution to the other area. In contrast Section 3 is much more $K$-theoretical in nature and uses more powerfull techniques proper to this area.

During the final stage of writing of the present paper our good friend and collegue Michel Matthey tragically died. We dedicate this paper to his memory, to his enthousiasm for sharing his ideas and his love for mathematics. 


\section{The proofs}

We give here the proofs of Theorems 1.1, 1.4, 1.8 and 1.13. But first we present a recollection of standard results from the topology and geometry of 3-manifolds. As general references on the subject, let us cite [19], [48], and also [2], [10], [31], [50].

A 3-manifold $M$ is called prime if it admits no non-trivial connected sum decomposition, i.e. if $M \approx M^{\prime} \# M^{\prime \prime}$, then at least one of $M^{\prime}$ and $M^{\prime \prime}$ is diffeomorphic to $S^{3}$. The manifold $M$ is said to be irreducible (in the sense of Hempel [19, p. 28]) if every embedded 2-sphere in $M$ bounds an embedded compact 3-ball. By [19, Lem. 3.13] a prime 3-manifold is either an $S^{2}$-bundle over $S^{1}$ or irreducible. In the later case, the homotopy exact sequence of the fiber sequence $S^{2} \rightarrow M \rightarrow S^{1}$ yields that $\pi_{1}(M)$ is infinite cyclic; if $M$ is orientable, then it is diffeomorphic to $S^{1} \times S^{2}$.

To begin our discussion of the two-stage decomposition, we let $M$ be a compact connected 3-manifold (but not necessarily closed, i.e. the boundary $\partial M$ may be non-empty). By the Kneser Prime Decomposition Theorem (see [35], [40], or [19, Thm. 3.15] where the closedness and the orientability of $M$ are avoided, see pp. $24 \& 32$ therein), one can decompose $M$ as a finite connected sum of compact connected 3-manifolds, say

$$
M \approx M_{1} \# M_{2} \# \cdots \# M_{q},
$$

with each $M_{i}$ prime; we can (and will) further suppose that the decomposition is normal in the sense of [19, p. 34], i.e. some $M_{i}$ is diffeomorphic to $S^{1} \times S^{2}$ if and only if $M$ is orientable. In this case, the decomposition is unique (up to reordering and diffeomorphism), and, under the extra assumption that $M$ is orientable, each $M_{i}$ is orientable as well, see [19, Thm. 3.21] (see also [40] for the orientable case). Of course, by the van Kampen Theorem, the fundamental group of $M$ decomposes as a finite free product

$$
\pi_{1}(M) \cong \pi_{1}\left(M_{1}\right) * \pi_{1}\left(M_{2}\right) * \cdots * \pi_{1}\left(M_{q}\right) .
$$

Recall that each $M_{i}$ is either an $S^{2}$-bundle over $S^{1}$, or irreducible.

Now, we let $M$ be a compact connected 3-manifold. In the sequel, by a surface $\Sigma$, we mean a compact connected 2-dimensional manifold (with possibly non-empty boundary $\partial \Sigma$ ). Consider a surface $\Sigma$ that is either properly embedded in $M$, i.e. $\partial \Sigma=\Sigma \cap \partial M$ (transverse intersection), or embedded in $\partial M$; in case $\Sigma \subseteq \partial M$ (so that $\Sigma$ is closed), note that 'sliding' $\Sigma$ along a small collar neighbourhood inside $M$, which is a trivial half-line bundle, we get an isotopic properly embedded surface in $M$. The surface $\Sigma$ is called 2-sided if it is embedded in $\partial M$, or if it admits a tubular neighbourhood in $M$ which is a trivial line bundle. The surface $\Sigma$ is said to be incompressible inside $M$ if it is 2 -sided, 
not diffeomorphic to the 2-sphere nor to a disk, and if it is $\pi_{1}$-injective, in the sense that the inclusion $\Sigma \hookrightarrow M$ induces a monomorphism $\pi_{1}(\Sigma) \hookrightarrow \pi_{1}(M)$. A 3-manifold $M$ is called $P^{2}$-irreducible if it is irreducible and if it contains no embedded 2-sided real projective plane.

A compact connected 3-manifold $M$ is called Haken if it is $P^{2}$-irreducible and contains a properly embedded 2-sided incompressible surface ( $M$ is supposed to be orientable, this amounts to require $M$ to be irreducible and to contain a properly embedded incompressible orientable surface). By [19, Lem. 6.7 (i)], if the compact connected 3-manifold $M$ is orientable and if $\partial M$ is non-empty and does not only consist of a collection of 2 -spheres, then the group $H_{1}(M ; \mathbb{Z})$ is infinite, and in this case, [19, Lem. 6.6] shows that $M$ is Haken provided it is irreducible (the surface $F$ constructed in the proof therein is orientable). A compact connected 3-manifold $M$ is called torus-irreducible (or geometrically atoroidal) if every incompressible 2-torus in $M$ is isotopic to a boundary component of $M$.

For the general definition, that we will not need, of a Seifert 3-manifold, we refer to [48, pp. 428-429]; what we will however need is the following characterization due to Epstein [16] in the compact case: a compact 3-manifold $M$ is Seifert if it admits a foliation by circles. By [26, Thm. 9.2] (see also [31, Thm. 1.38]), a deep result, a prime compact 3-manifold $M$ with infinite fundamental group $\pi_{1}(M)$ is Seifert if and only if $\pi_{1}(M)$ contains an infinite cyclic normal subgroup, in which case, there exists a short exact sequence of groups

$$
1 \rightarrow \mathbb{Z} \longrightarrow \pi_{1}(M) \stackrel{p}{\longrightarrow} \Gamma \rightarrow 1,
$$

with $\Gamma$ standing for a discrete subgroup of the isometry group of either $S^{2}$ (the 'round' 2-sphere), of $\mathbb{R}^{2}$ (the flat Euclidean plane), or of $\mathcal{H}^{2}$ (the hyperbolic plane). This means that $\Gamma$ is a discrete subgroup of one of the following three Lie groups (each having exactly two connected components):

$$
\mathrm{O}(3), \quad \mathbb{R}^{2} \rtimes \mathrm{O}(2) \text { and } \mathrm{SO}(2,1) .
$$

It will be important for us to note that for any finite subgroup $H$ of $\Gamma$, its pre-image $p^{-1}(H)$ in $\pi_{1}(M)$ sits in a short exact sequence

$$
1 \rightarrow \mathbb{Z} \longrightarrow p^{-1}(H) \longrightarrow H \rightarrow 1,
$$

and is therefore virtually cyclic, in the sense that it contains a cyclic subgroup (here, infinite) of finite index.

Next, we include a short algebraic incursion. A graph of groups $\mathcal{G}$ is a non-empty graph $G_{\mathcal{G}}=\left(E_{\mathcal{G}}, V_{\mathcal{G}}\right)$ (possibly with loops, i.e. with edges incident to only one vertex, and simple, i.e. with at most one loop per vertex and at most one edge joining two distinct vertices) equipped with two families of

TOME $136-2008-\mathrm{N}^{\circ} 1$ 
groups $\left\{G_{e}^{\prime}\right\}_{e \in E_{\mathcal{G}}}$ and $\left\{G_{v}\right\}_{v \in V_{\mathcal{G}}}$ parameterized by the edge set $E_{\mathcal{G}}$ and the vertex set $V_{\mathcal{G}}$, respectively, and a family $\left\{\iota_{e, v}: G_{e}^{\prime} \hookrightarrow G_{v} \mid v \in e\right\}_{e \in E_{\mathcal{G}}}$ of injective group homomorphisms, one for each pair $(e, v) \in E_{\mathcal{G}} \times V_{\mathcal{G}}$ consisting of an edge and an adjacent vertex; the groups in $\left\{G_{e}^{\prime}\right\}_{e \in E_{\mathcal{G}}}$ and in $\left\{G_{v}\right\}_{v \in V_{\mathcal{G}}}$ are called the edge-groups and the vertex-groups of $\mathcal{G}$, respectively. If the graph of groups $\mathcal{G}$ is finite and connected (i.e. if $G_{\mathcal{G}}$ is a finite connected graph), its fundamental group $\pi_{1}(\mathcal{G})$ is a group defined, up to isomorphism, by a finite induction process mixing the groups $G_{v}$ and $G_{e}^{\prime}$, using the incidence relation of $G_{\mathcal{G}}$ and the maps $\iota_{e, v}$, via amalgamated free products and HNN-extensions (see [49, Section 5] for details). The group $\pi_{1}(\mathcal{G})$ acts simplicially on the graph $G_{\mathcal{G}}$, with, up to isomorphism, vertex-stabilizers $\left\{G_{v}\right\}_{v \in V_{\mathcal{G}}}$ and edge-stabilizers $\left\{G_{e}^{\prime}\right\}_{e \in E_{\mathcal{G}}}$.

After Kneser's decomposition (or "sphere decomposition"), there is a second decomposition that we will need, namely the so-called JSJ-decomposition (or "torus decomposition"), named after Jaco-Shalen [25] and Johannson [27]. So, we let $M$ be an irreducible closed connected orientable 3-manifold. Then, there is a minimal finite family $\left\{T_{j}\right\}_{j \in J}$ (possibly empty) of embedded disjoint incompressible 2-sided closed 2-tori that separates $M$ into a finite set $\left\{M_{k}\right\}_{k \in K}$ of irreducible compact connected orientable 3-manifolds, each of which is either Seifert or torus-irreducible, possibly both. (Such a family is, up to isotopy inside $M$, unique; the finite index-sets $J$ and $K$ verify $|K|=|J|+1$.) Let us now describe the fundamental group of $M$ using a graph of groups. It turns out that there is a graph of groups $\mathcal{G}=\mathcal{G}_{M}$ with $E_{\mathcal{G}}=J$ and $V_{\mathcal{G}}=K$, and, for $j \in J$ and $k \in K, G_{j}^{\prime}=\pi_{1}\left(T_{j}\right) \cong \mathbb{Z}^{2}, G_{k}=\pi_{1}\left(M_{k}\right)$ and $\iota_{j, k}=\pi_{1}\left(\operatorname{incl}: T_{j} \hookrightarrow M_{k}\right)$, and with the incidence relation dictated by the combinatorial configuration of the separating family of tori; moreover (and most importantly), there is an isomorphism $\pi_{1}(M) \cong \pi_{1}(\mathcal{G})$. Indeed, this last property follows inductively from the van Kampen Theorem.

We also recall that an $n$-manifold $M$, possibly with non-empty boundary, is called hyperbolizable if its geometric interior $M \backslash \partial M$ admits a complete Riemannian metric for which the sectional curvature is constant with value -1 . In this case, $\pi_{1}(M) \cong \pi_{1}(M \backslash \partial M)$ is isomorphic to a discrete subgroup of the Lie group $\mathrm{SO}(n, 1)$ (and not necessarily of its identity component $\mathrm{SO}(n, 1)$ 。).

REMARK 2.1. - Suppose given a closed connected orientable 3-manifold $M$, and apply to it the following two-stage decomposition (without necessity of first capping $M$ off). First perform Kneser's prime decomposition; this produces finitely many pieces which are either $S^{1} \times S^{2}$ or closed irreducible manifolds. To each of the latter ones apply the JSJ-decomposition. The Thurston Geometrization Conjecture is the statement that the final pieces all have a (necessary unique) geometric structure among a list of eight possible ones (in a precise and specific sense, see [48], [50]). It might well happen that one has 
no decomposition to perform, for instance if one starts with $S^{3}$. The Thurston Geometrization Conjecture is known in all but two cases:

(a) for closed irreducible manifolds with finite fundamental group; this special case is known as the Thurston Elliptization Conjecture (which is equivalent to the combination of the Poincaré Conjecture and of the Spherical Space Form Conjecture);

(b) for closed, irreducible, non-Haken and non-Seifert manifolds with infinite fundamental group; in this case the manifold should be hyperbolizable: this is the content of the Thurston Hyperbolization Conjecture.

There is also a more general version of the Thurston Geometrization Conjecture (that we will not need and which is more technical to state), namely for connected orientable 3-manifolds that are compact (indeed, not necessarily closed). It is now known to hold in all cases, except for the very same two 'closed cases' (a) and (b).

Notice that in these cases no non-trivial decomposition is possible along a sub-manifold of codimension 1 . In case (a) because the fundamental group is too small for the manifold to contain an incompressible sub-surface of positive genus, in case (b) because of the "non-Haken" assumption.

For the proof of Theorem 1.1, we will also need the following result.

Proposition 2.2. - Let $M$ be a 3-manifold. Then, there exists a family $\left\{M_{n}\right\}_{n \in \mathbb{N}}$ of compact connected 3-manifolds and a family $\left\{f_{n}: M_{n} \rightarrow M\right\}_{n \in \mathbb{N}}$ of smooth immersions, such that each immersion $f_{n}$ induces an injective group homomorphism $\pi_{1}\left(M_{n}\right) \hookrightarrow \pi_{1}(M)$, and such that the fundamental group of $M$ is the union of (the images of) the fundamental groups of the members of the family, i.e.

$$
\pi_{1}(M)=\bigcup_{n \in \mathbb{N}} \pi_{1}\left(M_{n}\right) .
$$

Moreover, if $M$ is orientable, then one can further require the $M_{n}$ 's to be orientable.

Proof. - First, the group $\pi_{1}(M)$ being countable, we choose a sequence $\left(g_{n}\right)_{n \in \mathbb{N}}$ of elements of $\pi_{1}(M)$ (possibly with repetitions) such that the set $\left\{g_{n}\right\}_{n \in \mathbb{N}}$ generates $\pi_{1}(M)$. For each $n \in \mathbb{N}$, let $G_{n}:=\left\langle g_{1}, \ldots, g_{n}\right\rangle$ be the subgroup of $\pi_{1}(M)$ generated by $g_{1}, \ldots, g_{n}$. Since $G_{n}$ is finitely generated, by [19, Thm. 8.2], it is even finitely presented. Therefore, applying [19, Thm.8.1] (a result due to Jaco [24]), we can find a compact connected 3-manifold $M_{n}$ and an immersion $f_{n}: M_{n} \rightarrow M$ such that $\left(f_{n}\right)_{*}: \pi_{1}\left(M_{n}\right) \hookrightarrow \pi_{1}(M)$ is injective, as indicated, with image $G_{n}$ (note that one can indeed suppose each $M_{n}$ connected). The equality $\pi_{1}(M)=\bigcup_{n \in \mathbb{N}} \pi_{1}\left(M_{n}\right)$ is now obvious. Finally, for each $n, M_{n}$ being of the 
same dimension as $M$, and an immersion being a local homeomorphism, [14, Ex. 3 of VIII.2.22] applies to $f_{n}$ to show orientability of $M_{n}$ in case $M$ itself is orientable (note that [14, Prop. VIII.2.19] allows to incorporate successfully the case where $M_{n}$ and/or $M$ have a boundary).

Finally, we are in position to pass to the proofs of our theorems (in disorder).

Proof. - OfTheorem 1.4 Clearly, for the proofs, we can suppose that the compact orientable 3 -manifold $M$ we consider is connected, and that $M$ is cappedoff, i.e. that $M=\widehat{M}$. Let $G$ be the fundamental group of $M$. From the Kneser Prime Decomposition Theorem, we have deduced a finite free product decomposition

$$
G \cong \pi_{1}\left(M_{1}\right) * \pi_{1}\left(M_{2}\right) * \cdots * \pi_{1}\left(M_{q}\right) .
$$

Since the Baum-Connes Conjecture With Coefficients is stable under forming finite free products (see [43], [45]), if each $\pi_{1}\left(M_{i}\right)$ verifies this conjecture, then the same holds for $G$. Since $\pi_{1}\left(S^{1} \times S^{2}\right)$ is infinite cyclic, and since the BaumConnes Conjecture With Coefficients holds for the group $\mathbb{Z}$ (in fact, for any countable amenable group, including all abelian groups, see [21], [22]), we can now suppose further that $M$ is irreducible. As we have explained, if $M=\widehat{M}$ is not closed, i.e. if $\partial M \neq \varnothing$, then $M$ is Haken. In this case, by [45], or [8], or [51], its fundamental group satisfies the Baum-Connes Conjecture With Coefficients (the proof is based on the fact that a Haken manifold admits a so-called $h i$ erarchy in the sense of [19, p. 140] and on the results on graphs of groups we have recalled earlier). So, we are reduced to the case where $M$ is an irreducible closed connected orientable 3-manifold.

Now, we apply to $M$ a JSJ-decomposition. Earlier, in such a situation, $\pi_{1}(M)$ has been expressed using a certain graph of groups. By [45] again, the Baum-Connes Conjecture With Coefficients (and also the plain Baum-Connes Conjecture, see [41, Thm. 5.13 in Part I]) is stable under taking finite connected graphs of groups, i.e. if a finite connected graph of groups $\mathcal{G}$ has all its edgegroups $\left\{G_{e}^{\prime}\right\}_{e \in E_{\mathcal{G}}}$ and vertex-groups $\left\{G_{v}\right\}_{v \in V_{\mathcal{G}}}$ satisfying the Baum-Connes Conjecture (resp. With Coefficients), then so does its fundamental group $\pi_{1}(\mathcal{G})$. As, in our case, the edge-groups are isomorphic to the abelian group $\mathbb{Z}^{2}$, the Baum-Connes Conjecture With Coefficients holds for them. So, it remains to deal with the vertex-groups. These are fundamental groups of compact connected 3-manifolds, each of which is either Seifert or torus-irreducible, possibly both. We distinguish three cases for each of these pieces, that we call, say, $N$.

1 ) If $N$ is Seifert, then, as we have seen, $\pi_{1}(N)$ sits in a short exact sequence

$$
1 \rightarrow \mathbb{Z} \longrightarrow \pi_{1}(N) \stackrel{p}{\longrightarrow} \Gamma \rightarrow 1,
$$


with $\Gamma$ a discrete subgroup of one of the Lie groups $\mathrm{O}(3), \mathbb{R}^{2} \rtimes \mathrm{O}(2)$ and $\mathrm{SO}(2,1)$, which are almost connected, i.e. they have finitely many connected components (here, exactly 2). Now, consider the following facts concerning $\Gamma$ :

(i) If $\Gamma$ is a discrete subgroup of the compact group $\mathrm{O}(3)$, then $\Gamma$ is finite and thus satisfies the Baum-Connes Conjecture With Coefficients (see [29]).

(ii) The so-called Kasparov $\gamma$-element is equal to one for both Lie groups $\mathrm{SO}(2,1)$ and $\mathbb{R}^{2} \rtimes \mathrm{O}(2)$. Since any discrete subgroup of an almost connected Lie group with $\gamma=1$ satisfies the Baum-Connes Conjecture With Coefficients, so does $\Gamma$. Given $n \geq 2$, for $\operatorname{SO}(n, 1)$, the equality $\gamma=1$ is established in [32], and for $\mathbb{R}^{n} \rtimes \mathrm{O}(n)$, the $\gamma$-element, being invariant under group retractions (see [32]), is the image of the $\gamma$-element of $\mathrm{O}(n)$, which, by a computation carried out in [4], is equal to one as well. It could also be said that if $\Gamma$ is a discrete subgroup of $\operatorname{SO}(2,1)$ or of $\mathbb{R}^{2} \rtimes \mathrm{O}(2)$, then $\Gamma$ has the Haagerup property (see [11, Thm. 4.0.1\& Prop. 6.1.5] for $\Gamma \subset \mathrm{SO}(2,1)$, and [9] for $\Gamma \subset \mathbb{R}^{2} \rtimes \mathrm{O}(2)$, in which case $\Gamma$ is amenable) and then conclude by [21], [22].

We have also seen that for any finite subgroup $H$ of $\Gamma$, the pre-image $p^{-1}(H)$ inside $\pi_{1}(N)$ is virtually cyclic and therefore amenable (since the class of discrete amenable groups contains abelian groups and finite groups, and is stable under taking group extensions). By [21], [22] again, each $p^{-1}(H)$ satisfies the Baum-Connes Conjecture With Coefficients; by [44], this is enough to guarantee that $\pi_{1}(N)$ itself satisfies this conjecture. This is it for case 1 ).

2) If $N$ has finite fundamental group (hence $N$ is non-Seifert and, in fact, torus-irreducible), then the Baum-Connes Conjecture With Coefficients is known for the finite group $\pi_{1}(N)$, as we have already said (see [29]).

3 ) If $N$ is non-Seifert with infinite fundamental group (and $N$ is then torusirreducible), then, we distinguish four non mutually excluding sub-cases.

(i) If $N$ is Haken, then, by [45], or [8], or [51], its fundamental group satisfies the Baum-Connes Conjecture With Coefficients.

(ii) If $N$ is hyperbolizable, then, as recalled earlier, $\pi_{1}(N)$ is a discrete subgroup of $\mathrm{SO}(3,1)$. As seen in 1) (ii) above, such a discrete subgroup satisfies the Baum-Connes Conjecture With Coefficients.

(iii) If $N$ (which is non-Seifert and has infinite fundamental group) is neither Haken, nor hyperbolizable, then our technical hypothesis in the statement of the theorem precisely guarantees that $\pi_{1}(N)$ also satisfies this conjecture.

This completes our discussion of case 3 ).

We conclude, for each considered piece $N$ obtained after the JSJdecomposition, that, in any of these three events 1)-3), the group $\pi_{1}(N)$ 
satisfies the Baum-Connes Conjecture With Coefficients, and consequently that so does $\pi_{1}(M)$.

Proof of Theorem 1.1. - By [7, Thm. 1.1], if a countable discrete group $G$ is the union $G=\bigcup_{n \in \mathbb{N}} G_{n}$ of a collection of subgroups all satisfying the BaumConnes Conjecture With Coefficients, then so does $G$. Since the fundamental group of a compact manifold is countable (at most), combining this with Proposition 2.2, the result follows directly from Theorem 1.4; indeed, as we have recalled, the Thurston Geometrization Conjecture implies the Thurston Hyperbolization Conjecture, which precisely predicts that each piece obtained exactly after the second stage of the two-stage decomposition of the statement and which is non-Seifert, non-Haken and has infinite fundamental group is hyperbolizable.

Proof of Theorem 1.8. - We may suppose that $M$ is connected and cappedoff, so that $M=\widehat{M}$. Using Kneser's (normal) prime decomposition, we can write $M$ as

$$
M \approx M_{1} \# \cdots \# M_{p} \# M_{p+1} \# \cdots \# M_{q}
$$

with $M_{1}, \ldots, M_{q}$ denoting prime compact connected 3-manifolds (possibly nonorientable), where $M_{1}, \ldots, M_{p}$ are irreducible and $M_{p+1}, \ldots, M_{q}$ are prime but not irreducible. Therefore, $M_{p+1}, \ldots, M_{q}$ are $S^{2}$-bundles over $S^{1}$ and have consequently an infinite cyclic fundamental group, and hence verifying the BaumConnes Conjecture With Coefficients. Now, fix $i \in\{1, \ldots, p\}$. By assumption, either $M_{i}$ is orientable and Theorem 1.4 applies to it to show that it satisfies the Baum-Connes Conjecture With Coefficients, or $N:=M_{i}$ is an irreducible, nonorientable, compact, connected and capped-off 3-manifold having either infinite cyclic fundamental group, or having no 2-torsion in its fundamental group and with each component of $\partial M$ incompressible in $M$ (possibly with $\partial M=\varnothing$ ). Let us now deal with $N$. If $\pi_{1}(N) \cong \mathbb{Z}$ then, once again, $N$ satisfies the Baum-Connes Conjecture. So, we suppose that $\pi_{1}(N)$ is 2-torsion-free, but not infinite cyclic. By Kneser's Conjecture on free products, proved for instance in [19, Thm. 7.1], since $N$ is irreducible, its fundamental group $\pi_{1}(N)$ is indecomposable with respect to free products. This property, together with the fact that $\pi_{1}(N)$ is not infinite cyclic and does not contain 2-torsion, implies that [19, Lem. 10.1] applies to $N$, which is capped-off. The conclusion of this result is that $N$ is $P^{2}$-irreducible (in the notation of [19, Lem. 10.1], since $N$ is irreducible and non-orientable, we can take $N$ as $\mathcal{P}(N)$ and the occurring homotopy sphere is diffeomorphic to $S^{3}$ ). Combining [19, Lem. 6.7 (ii) \& Lem. 6.6] for the $P^{2}$-irreducible manifold $N$, we obtain, inside $N$, a properly embedded, 2 -sided incompressible surface $\Sigma$, which is non-separating. (In particular, $N$ is Haken.) 
Therefore, cutting $N$ along $\Sigma$, we get a compact connected $P^{2}$-irreducible manifold $N^{\prime}$ with non-empty boundary. Invoking [19, Thm. 13.3], we obtain a hierarchy for $N^{\prime}$ (see details in [19, p. 140]). Consequently, the argument given in [45] proves that the group $\pi_{1}\left(N^{\prime}\right)$ satisfies the Baum-Connes Conjecture With Coefficients. Now, there is an isomorphism $\pi_{1}(N) \cong \pi_{1}\left(N^{\prime}\right) *_{\pi_{1}(\Sigma)}$, i.e. $\pi_{1}(N)$ is an HNN-extension with base $\pi_{1}\left(N^{\prime}\right)$ and over the surface group $\pi_{1}(\Sigma)$. Fundamental groups of closed surfaces (orientable or not) are one-relator groups, so that, by [45], they verify the Baum-Connes Conjecture With Coefficients. By [45] once again, this conjecture is stable under forming HNN-extensions, so that the conjecture holds for $\pi_{1}(N)$ too. In total, we see that each "free factor" in the initial decomposition

$$
\pi_{1}(M) \cong \pi_{1}\left(M_{1}\right) * \cdots * \pi_{1}\left(M_{p}\right) * \pi_{1}\left(M_{p+1}\right) * \cdots * \pi_{1}\left(M_{q}\right)
$$

satisfies the conjecture, hence also their finite free product $\pi_{1}(M)$, still by [45].

Proof of Theorem 1.13. - It is standard that surjectivity of the Baum-Connes assembly map (in degree 0) for a torsion-free discrete group $G$ implies the Kadison-Kaplansky Conjecture for $G$, and hence Kaplansky's Idempotent Conjecture for $G$ since $\mathbb{C} G$ is a sub-algebra of $C_{r}^{*} G$ (see for instance [41, Lem. 7.2 in Part I] or [47, Section 5] for a proof). So, the first part of Theorem 1.13 follows directly from Theorem 1.1. For the second part, suppose that $G=\pi_{1}(M)$, where $M$ is a connected orientable 3-manifold decomposed as

$$
M \approx M_{1} \# M_{2} \# \cdots \# M_{q}
$$

with each $M_{i}$ a compact connected orientable prime 3 -manifold with, by assumption, infinite fundamental group. By [19, Thm.9.8] (see also p. 170 therein), each fundamental group $\pi_{1}\left(M_{i}\right)$ is torsion-free, hence also the finite free product $G \cong \pi_{1}\left(M_{1}\right) * \pi_{1}\left(M_{2}\right) * \cdots * \pi_{1}\left(M_{q}\right)$. Consequently, the first part of the theorem applies to $G$.

\section{The class $\mathcal{C}_{3}$}

As we have seen in the proof of Theorem 1.4, the JSJ decomposition of a manifold presents its fundamental group naturally as a (finite) graph of groups. The key point of the proof was then to show that the Baum-Connes conjecture for the fundamental group is inherited from the vertex and edge groups. This leads naturally to consider a class of groups that is "hereditary with respect to actions on oriented trees with finite fundamental domain", i.e. whenever a group, say $G$, has such an action and moreover the stabilizers of the vertices and of the edges belong to the class then so does the group $G$. 
Definition 3.1. - The class $\mathcal{C}_{3}$ is the smallest class of groups that is

i) stable by isomorphism;

ii) contains all finite groups;

iii) contains all fundamental groups of compact Seifert manifolds;

iv) contains all cocompact torsion free subgroups of isometries of the hyperbolic 3 -space $\mathcal{H}_{3}$

v) is hereditary with respect to actions on oriented trees with finite fundamental domain.

According to the results of the previous section, if the Thurston hyperbolization conjecture holds, then the class $\mathcal{C}_{3}$ contains all fundamental groups of compact oriented 3-manifolds. Moreover by the proof of Theorem 1.1, the elements of $\mathcal{C}_{3}$ satisfy the Baum-Connes conjecture. We prove in this section some further properties for groups in this class, namely property $\left(\mathrm{BC}^{\prime}\right)$ and exactness.

In Section 1, when we defined the Baum-Connes assembly map, we pointed out the fact that this morphism should be valued in the $K$-theory of the reduced $C^{*}$-algebra (or more generally in the reduced crossed product algebra), and that Kazhdan property $(\mathrm{T})$ is an obstruction for surjectivity of Miščenko morphism. We shall see that this obstruction do not occure for a group in the class $\mathcal{C}_{3}$ since these groups are $K$-amenable [12], i.e the morphism $\lambda^{G}: C^{*}(G) \rightarrow C_{r}^{*}(G)$ induced an equivalence in $K K\left(C^{*}(G), C_{r}^{*}(G)\right)$. This $K$-amenability property in particular implies for a discrete group $G$ that

$$
\lambda_{*}^{G}: K_{*}^{\mathrm{top}}\left(C_{r}^{*}(G)\right) \longrightarrow K_{*}^{\mathrm{top}}\left(C^{*}(G)\right)
$$

is an isomorphism and more generally that the $K$-theory of reduced and maximal crossed products algebras should be isomorphic. In consequence, since the only $K$-amenable groups having Kazhdan property $(\mathrm{T})$ are finite group, we recover in this way the following result of [17]: if the Thurston hyperbolization conjecture holds, then the fundamental groups of a compact oriented 3manifolds has Kazhdan property ( $\mathrm{T}$ ) if and only if it is finite.

3.1. Property $\left(\mathrm{BC}^{\prime}\right)$. - Let $G$ be a discrete group and let $X$ be a locally compact space equipped with a proper $G$-action. Recall that a $G \ltimes X$-algebra is a $C^{*}$-algebra equipped with an action of $G$ by automorphisms and with an $G$-equivariant morphism $\Phi: C_{0}(X) \longrightarrow \mathcal{Z}(M(A))(\mathcal{Z}(M(A))$ being the center of the multipliers algebra of $A$ ) such that $\Phi\left(C_{0}(X)\right) \cdot A$ is dense in $A$. A $G$ $C^{*}$-algebra is call proper if there exists a $G$-proper action on a locally compact space $X$ such that $A$ is a $G \ltimes X$-algebra. 
Definition 3.2 (see [51]). - A group $G$ verifies property $\left(\mathrm{BC}^{\prime}\right)$ if it is contained in a discrete group $G^{\prime}$ such that there exists a proper $G^{\prime}$ compact space $X$, a $G^{\prime} \ltimes X$-algebra $\mathcal{A}$ and elements $\alpha \in K K^{G^{\prime}}(\mathbb{C}, \mathcal{A})$ and $\beta \in K K^{G^{\prime}}(\mathcal{A}, \mathbb{C})$ such that $\alpha \otimes_{\mathcal{A}} \beta=1$ in $K K^{G^{\prime}}(\mathbb{C}, \mathbb{C})$.

Theorem 3.3 (see [51]). - If a group $G$ satifies property $\left(\mathrm{BC}^{\prime}\right)$, then

1) G satisfies the Baum-Connes conjecture;

2) $G$ is $K$-amenable.

3) G satisfies the Künneth formula and the Universal Coefficients Theorem.

Theorem 3.4 (see [51]). - Property $\left(\mathrm{BC}^{\prime}\right)$ is hereditary under finite fundamental domain action on trees.

Since the isometry group of $\mathcal{H}_{3}$ has a $\gamma$-element in the sense of Kasparov equal to 1 [32], every torsion free cocompact discrete subgroup of isometries of $\mathcal{H}_{3}$ satisfies property $(B C)$. Moreover, as we shall see later in subsection 3.3, fundamental groups of prime compact Seifert manifolds have property $\left(\mathrm{BC}^{\prime}\right)$. Thus we obtain:

THEOREM 3.5. - Every group in the class $\mathcal{C}_{3}$ satisfies the property $\left(\mathrm{BC}^{\prime}\right)$ and in particular, if the Thurston hyperbolization conjecture holds, then fundamental groups of compact oriented manifolds have property $\left(\mathrm{BC}^{\prime}\right)$.

It is a well known that property $\left(\mathrm{BC}^{\prime}\right)$ for discrete groups implies the socalled Bost Conjecture: Let $G$ be a discrete group, then the inclusion

$$
i: \ell^{1}(G) \hookrightarrow C_{r}^{*} G
$$

induces an isomorphism

$$
i_{*}: K_{*}^{\mathrm{top}}\left(\ell^{1}(G)\right) \stackrel{\cong}{\longrightarrow} K_{*}^{\mathrm{top}}\left(C_{r}^{*} G\right) .
$$

We give for the benefit of the reader a proof of this fact. If $\mathcal{A}$ is a $G$ - $C^{*}$-algebra, we define

$$
\ell^{1}(G, \mathcal{A}):=\left\{f: G \rightarrow \mathcal{A} ; \sum_{g \in G}\|f(g)\|<+\infty\right\},
$$

equipped with the convolution product

$$
f * h(g)=\sum_{g^{\prime} \in G} f\left(g^{\prime}\right) g^{\prime}\left(h\left(g^{\prime-1} g\right)\right)
$$

and the norm $\|f\|=\sum_{g \in G}\|f(g)\|$. Then $\ell^{1}(G, \mathcal{A})$ is a dense subalgebra of the reduced crossed product $\mathcal{A} \rtimes_{\text {red }} G$. The following lemma is proved in [36, Section 1. 7] 
Lemma 3.6. - If $\mathcal{A}$ is a proper $G$ - $C^{*}$-algebra, the inclusion

$$
i: \ell^{1}(G, \mathcal{A}) \longleftrightarrow \mathcal{A} \rtimes_{\text {red }} G
$$

induces an isomorphism

$$
i_{*}: K_{*}^{\mathrm{top}}\left(\ell^{1}(G, \mathcal{A})\right) \stackrel{\cong}{\longrightarrow} K_{*}^{\mathrm{top}}\left(\mathcal{A} \rtimes_{\text {red }} G\right) .
$$

The proof of the Bost conjecture for groups with property $\left(\mathrm{BC}^{\prime}\right)$ is now a standard argument using the $\gamma$-element trick. Let $X$ be a proper $G$-space, let $\mathcal{A}$ be a $G \ltimes X$-algebra and let $\alpha \in K K^{G}(\mathbb{C}, \mathcal{A})$ and $\beta \in K K^{G}(\mathcal{A}, \mathbb{C})$ be elements such that $\alpha \otimes_{\mathcal{A}} \beta=1$ in $K K^{G}(\mathbb{C}, \mathbb{C})$. By [36, Section 1.7], the following diagram is commutative

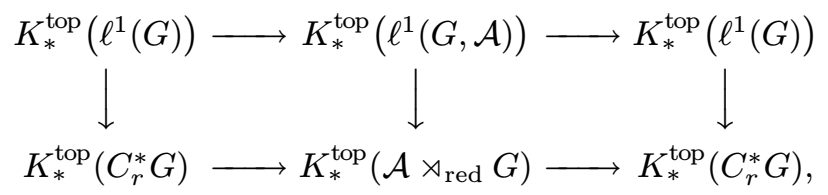

where

- the vertical arrows are induced by the inclusions

$$
\ell^{1}(G) \hookrightarrow C_{r}^{*} G \quad \text { and } \quad \ell^{1}(G, \mathcal{A}) \hookrightarrow \mathcal{A} \rtimes_{\text {red }} G ;
$$

- the bottom arrows are the right Kasparov products by elements induced by $\alpha$ and $\beta$ [33];

- the top arrows are induced by elements of Lafforgue bivariant $K$-theory for Banach algebras coresponding to $\alpha$ and $\beta$ [36].

Since $\alpha \otimes_{A} \beta=1$, the horizontal compositions are the identity [33], [36] and therefore the morphism $K_{*}^{\text {top }}\left(\ell^{1}(G)\right) \longrightarrow K_{*}^{\text {top }}\left(C_{r}^{*} G\right)$ is a direct factor of the morphism $K_{*}^{\text {top }}\left(\ell^{1}(G, \mathcal{A})\right) \longrightarrow K_{*}^{\text {top }}\left(\mathcal{A} \rtimes_{\text {red }} G\right)$ which is an isomorphism by Lemma 3.6. This guarantees that $K_{*}^{\text {top }}\left(\ell^{1}(G)\right) \longrightarrow K_{*}^{\text {top }}\left(C_{r}^{*} G\right)$ is an isomorphism.

3.2. Exactness. - In this subsection, all $C^{*}$-algebras are assumed to be separable.

Definition 3.7. - A discrete group $G$ is called exact if every short exact sequence

$$
0 \rightarrow \mathcal{J} \longrightarrow \mathcal{A} \longrightarrow \mathcal{A} / \mathcal{J} \rightarrow 0
$$

of $G-C^{*}$-algebras induces a short exact sequence for reduced crossed-products

$$
0 \rightarrow \mathcal{J} \rtimes_{r} G \longrightarrow \mathcal{A} \rtimes_{r} G \longrightarrow(\mathcal{A} / \mathcal{J}) \rtimes_{r} G \rightarrow 0 .
$$

Let us check that $\mathcal{C}_{3}$-class groups are exact. Exactness enjoys the following stability properties: 
TheOREM 3.8 (see [15]). — Exactness is stable by amalgamated product for discrete groups.

HNN extensions are made out of amalgamated products and semi-direct products by $\mathbb{Z}$. Since exactness is stable under extensions [34], HNN extensions of exact groups are exact. Hence we obtain :

Corollary 3.9. - Exactness is hereditary under finite fundamental domain action on trees.

All discrete subgroups of Lie groups with finitely many components are exact (see [34]), thus discrete subgroups of $\mathcal{H}_{3}$ are exact. We shall see in Subsection 3.3 that fundamental group of prime compact Seifert manifolds are exact. Hence we have

THEOREM 3.10. - Every element in the class $\mathcal{C}_{3}$ is exact and in particular, if the Thurston hyperbolization conjecture holds, then the fundamental group of a compact oriented manifold is exact.

We end this section by quoting the characterization of exactness in terms of amenability at infinity [1], [46].

Definition 3.11. - Let $G$ be a discrete group and let us denote by $\operatorname{Prob}(G)$ the set of Borel probility on $G$, equipped with the weak ${ }^{*}$-topology. The group $G$ is said to be amenable at infinity if there is a second countable compact $G$-space $X$ and a sequence $\left(\mu_{n}\right)_{n \in \mathbb{N}}$ of continuous functions

$$
\mu_{n}: X \longrightarrow \operatorname{Prob}(G)
$$

such that $\lim _{n \rightarrow \infty} g \mu_{n}-\mu_{n}=0$ for every $g \in G$.

TheOREM 3.12. - A discret group $G$ is exact if and only if it is amenable at infinity

Finally, let us recall that the Baum-Connes assembly map for a group that is amenable at infinity is split injective [20].

TOME $136-2008-\mathrm{N}^{\mathrm{O}} 1$ 
3.3. Fundamental groups of prime compact Seifert manifolds. - As we have seen before, if $M$ is a prime compact Seifert manifold, then there exists a short exact sequence of groups

$$
1 \rightarrow \mathbb{Z} \longrightarrow \pi_{1}(M) \stackrel{p}{\longrightarrow} \Gamma \rightarrow 1,
$$

with $\Gamma$ standing for a discrete subgroup of one of the following three Lie groups:

$$
\mathrm{O}(3), \quad \mathbb{R}^{2} \rtimes \mathrm{O}(2) \text { and } \mathrm{SO}(2,1) .
$$

Since discrete subgroups of Lie groups with finitely many connected components are exact and exactness is stable under extensions [34], we get that $\pi_{1}(M)$ is exact. Let us check now that fundamental groups of irreducible Seifert manifolds satisfies property $\left(\mathrm{BC}^{\prime}\right)$.

Assume first that $M$ has no boundary. Then the universal covering space $\widetilde{M}$ of $M$ admits one of these three possible geometries

1) $\mathbb{R}^{3}$;

2) $\mathcal{H}_{2} \times \mathbb{R}$, where $\mathcal{H}_{2}$ is the hyperbolic half-space ;

3) $\widetilde{\mathrm{SL}}_{2}(\mathbb{R})$ the universal covering space of $\mathrm{SL}_{2}(\mathbb{R})$.

LEMMA 3.13. - If $M$ is a prime compact Seifert manifold without boundary, then $\pi_{1}(M)$ has the property $\left(\mathrm{BC}^{\prime}\right)$.

Proof. - The isometry group of any of this three geometries as $\gamma$-element in the sense of Kasparov equal to 1 (see [32]); notice that $\widetilde{S L}_{2}(\mathbb{R}$ ) is locally isomorphic to $\mathrm{SO}(3,1)$. Since $\pi_{1}(M)$ is cocompact in the suitable isometry group, it satisfies property $\left(\mathrm{BC}^{\prime}\right)$.

If $M$ has boundary, then $\Gamma$ is a (finite) free product of a finitely generated free group and of some cyclic finite groups. Let $T$ be the tree associated to this free product. The action of $\Gamma$ on $T$ has finite fundamental domain and the vertices stabilizer are either free groups or finite cyclic groups. So the group $\pi_{1}(M)$ acts through $\Gamma$ on the tree associated to this free product $T$ with finite fundamental domain and the vertices stabilizers are extensions of $\mathbb{Z}$ either by a finitely generated free group or by a finite cyclic group. Since property $\left(\mathrm{BC}^{\prime}\right)$ is heriditary under finite fundamental domain action on trees, the two following lemmas show that $\pi_{1}(M)$ satisfies property $\left(\mathrm{BC}^{\prime}\right)$.

LEMma 3.14. - Let $\Gamma$ be a discrete group fitting in a short exact sequence

$$
1 \rightarrow \mathbb{Z} \longrightarrow \Gamma \longrightarrow \mathbb{F}_{n} \rightarrow 1,
$$

where $\mathbb{F}_{n}$ denotes the free group on $n$ generators. Then $\Gamma$ satisfies property $\left(\mathrm{BC}^{\prime}\right)$. 
Proof. - The group $\Gamma$ acts via $\mathbb{F}_{n}$ on its associated tree (its Cayley diagram). The stabilizer of vertices are isomorphic to $\mathbb{Z}$ which satisfies property $\left(\mathrm{BC}^{\prime}\right)$ and thus $\Gamma$ satisfies also property $\left(\mathrm{BC}^{\prime}\right)$.

Lemma 3.15. - Let $\Gamma$ be a discrete group fitting in a short exact sequence

$$
1 \rightarrow \mathbb{Z} \longrightarrow \Gamma \longrightarrow \mathbb{Z}_{n} \rightarrow 1
$$

where $\mathbb{Z}_{n}$ is the cyclic group of order $n$. Then $\Gamma$ satisfies property $\left(\mathrm{BC}^{\prime}\right)$.

Proof. — The $\mathbb{Z}$-valued cocycle associated to the short exact sequence

$$
1 \rightarrow \mathbb{Z} \longrightarrow \Gamma \longrightarrow \mathbb{Z}_{n} \rightarrow 1
$$

extends to an $\mathbb{R}$-valued cocycle and thefore the short exact sequence lies in a short sequence

$$
1 \rightarrow \mathbb{R} \longrightarrow G \longrightarrow \mathbb{Z}_{n} \rightarrow 1 \text {. }
$$

The group $G$ is an amenable Lie group with finitely many connected components and thus, its $\gamma$-element in the sense of Kasparov is equal to 1 (see [33]). The group $\Gamma$ being cocompact in $G$, it satisfies property $\left(\mathrm{BC}^{\prime}\right)$.

\section{BIBLIOGRAPHY}

[1] C. Anantharaman-Delaroche - "Amenability and exactness for dynamical systems and their $C^{*}$-algebras", Trans. Amer. Math. Soc. 354 (2002), p. 4153-4178.

[2] M. T. ANDERSON - "Scalar curvature and geometrization conjectures for 3-manifolds", Math. Sci. Res. Inst. Publ. 30 (1997), p. 49-82.

[3] M. F. АтІуан - Elliptic operators, discrete groups and von Neumann algebras, Astérisque, vol. 32-33, Soc. Math. France, 1976.

[4] M. F. Атіyah \& I. M. Singer - "The index of elliptic operators. I", Ann. of Math. (2) 87 (1968), p. 484-530.

[5] P. BAum \& A. Connes - "Geometric $K$-theory for Lie groups and foliations", Enseign. Math. (2) 46 (2000), p. 3-42.

[6] P. Baum, A. Connes \& N. Higson - "Classifying space for proper actions and $K$-theory of group $C^{*}$-algebras", Contemp. Math. 167 (1994), p. 240-291.

[7] P. Baum, S. Millington \& R. Plymen - "Local-global principle for the Baum-Connes conjecture with coefficients", K-Theory 28 (2003), p. 1-18.

[8] C. Béguin, H. Bettaieb \& A. Valette - " $K$-theory for $C^{*}$-algebras of one-relator groups", K-Theory 16 (1999), p. 277-298.

TOME $136-2008-\mathrm{N}^{\mathrm{O}} 1$ 
[9] M. E. B. BekKa, P.-A. Cherix \& A. Valette - "Proper affine isometric actions of amenable groups", in Novikov conjectures, index theorems and rigidity, Vol. 2 (Oberwolfach, 1993), London Math. Soc. Lecture Note Ser., vol. 227, Cambridge Univ. Press, 1995, p. 1-4.

[10] F. BonAHON - "Geometric structures on 3-manifolds", in Handbook of geometric topology (R. Daverman et al., eds.), Elsevier, 2002, p. 93-164.

[11] P.-A. Chérix, M. Cowling, P. Jolissaint, P. Julg \& A. Valette - Groups with the Haagerup property, Gromov's a-T-menability, Progress in Math., vol. 197, Birhäuser, 2001.

[12] J. Cuntz - "K-theoretic amenability for discrete groups", J. reine angew. Math. 344 (1983), p. 180-195.

[13] M. Denn - "Über die Topologie des dreidimensionalen Raumes", Math. Ann. 69 (1910), p. 137-168.

[14] A. Dold - "Lectures on algebraic topology", in Classics in Mathematics, Springer, 1972, Die Grundlehren der mathematischen Wissenschaften, Band 200, p. 377.

[15] K. J. DyKema - "Exactness of reduced amalgamated free product $C^{*}$ algebras", Forum Math. 16 (2004), p. 161-180.

[16] D. B. A. Epstein - "Periodic flows on 3-manifolds", Ann. of Math. 95 (1972), p. 66-82.

[17] K. Fujiwara - "3-manifold groups and property $T$ of Kazhdan", Proc. Japan Acad. Ser. A Math. Sci. 75 (1999), p. 103-104.

[18] M. Gromov - "Geometric reflections on the Novikov conjecture", in Novikov conjectures, index theorems and rigidity, Vol. 1 (Oberwolfach, 1993), London Math. Soc. Lecture Note Ser., vol. 226, Cambridge Univ. Press, 1995, p. 164-173.

[19] J. Hempel - 3-Manifolds, Annals of Math Studies, vol. 86, Princeton University Press, 1976, Ann. of Math. Studies, No. 86.

[20] N. Higson - "Bivariant $K$-theory and the Novikov conjecture", Geom. Funct. Anal. 10 (2000), p. 563-581.

[21] N. Higson \& G. KASPAROv - "Operator $K$-theory for groups which act properly and isometrically on Hilbert space", Electron. Res. Announc. Amer. Math. Soc. 3 (1997), p. 131-142.

[22] _ " $E$-theory and $K K$-theory for groups which act properly and isometrically on Hilbert space", Invent. Math. 144 (2001), p. 23-74.

[23] F. HiRzebruCH - Topological methods in algebraic geometry, Classics in Mathematics, Springer, 1995.

[24] W. H. JACO - "Finitely presented subgroups of three-manifold groups", Invent. Math. 13 (1971), p. 335-346. 
[25] W. H. Jaco \& P. B. Shalen - Seifert fibered spaces in 3-manifolds, Mem. Amer. Math. Soc., vol. 21, 1979.

[26] M. Jankins \& W. D. Neumann - "Lectures on Seifert manifolds", in Brandeis Lecture Notes, Brandeis Lecture Notes, vol. 2, Brandeis University, 1983.

[27] K. JohANNSON - "Homotopy equivalence of 3-manifolds with boundaries", in Springer Lecture Notes in Math., vol. 761, 1979.

[28] F. E. A. Johnson \& J. P. WAlton - "Parallelizable manifolds and the fundamental group", Mathematika 47 (2000), p. 165-172 (2002).

[29] P. JulG - "K-théorie équivariante et produits croisés", C. R. Acad. Sci. Paris Sér. I Math. 292 (1981), p. 629-632.

[30] P. Julg \& G. Kasparov - "Operator $K$-theory for the group $\mathrm{SU}(n, 1)$ ", J. reine angew. Math. 463 (1995), p. 99-152.

[31] M. KAPOVICH - Hyperbolic manifolds and discrete groups, Progress in Mathematics, vol. 183, Birkhäuser, 2001.

[32] G. KAsparov - "Lorentz groups: $K$-theory of unitary representations and crossed products", Dokl. Akad. Nauk SSSR 275 (1984), p. 541-545.

[33] _ "Equivariant $K K$-theory and the Novikov conjecture", Invent. Math. 91 (1988), p. 147-201.

[34] E. Kirchberg \& S. WASSERMANN - "Permanence properties of $C^{*}$-exact groups", Doc. Math. 4 (1999), p. 513-558.

[35] H. KNESER - "Geschlossene Flächen in dreidimensionalen Mannigfaltigkeiten", Jahresbericht der Deut. Math. Verein. 38 (1929), p. 248-260.

[36] V. LAfForgue - " $K$-théorie bivariante pour les algèbres de Banach et conjecture de Baum-Connes", Invent. Math. 149 (2002), p. 1-95.

[37] H. B. Lawson, JR. \& M.-L. Michelsohn - Spin geometry, Princeton Mathematical Series, vol. 38, Princeton University Press, 1989.

[38] A. MARKOV - "The insolubility of the problem of homeomorphy", Dokl. Akad. Nauk SSSR 121 (1958), p. 218-220.

[39] V. MATHAI - "The Novikov conjecture for low degree cohomology classes", Geom. Dedicata 99 (2003), p. 1-15.

[40] J. W. Milnor - "A unique factorization theorem for 3-manifolds", Amer. J. Math. 84 (1962), p. 1-7.

[41] G. Mislin \& A. VAlette - "Proper group actions and the Baum-Connes conjecture", in Advanced Course in Mathematics, CRM Barcelona, Advanced Courses in Mathematics. CRM Barcelona, Birkhäuser, 2003.

[42] A. S. MišČEnKo - "Infinite-dimensional representations of discrete groups, and higher signatures (Russian)", Izv. Akad. Nauk SSSR Ser. Mat. 38 (1974), p. 81-106. 
[43] H. Oyono-Oyono - "La conjecture de Baum-Connes pour les groupes agissant sur les arbres", C. R. Acad. Sci. Paris Sér. I Math. 326 (1998), p. $799-804$.

[44] _ "Baum-Connes conjecture and extensions", J. reine angew. Math. 532 (2001), p. 133-149.

[45] _ "Baum-Connes conjecture and group actions on trees", $K$-Theory 24 (2001), p. 115-134.

[46] N. OzawA - "Amenable actions and exactness for discrete groups", $C$. $R$. Acad. Sci. Paris Sér. I Math. 330 (2000), p. 691-695.

[47] M. Puschnigg - "The Kadison-Kaplansky conjecture for word-hyperbolic groups", Invent. Math. 149 (2002), p. 153-194.

[48] P. ScotT - "The geometries of 3-manifolds", Bull. London Math. Soc. 15 (1983), p. 401-487.

[49] J-P. SERRE - Arbres, amalgames, SL 2 , Astérisque, vol. 46, 1983.

[50] W. P. Thurston - Three-dimensional geometry and topology. Vol. 1, Princeton Mathematical Series, vol. 35, Princeton University Press, 1997, Edited by Silvio Levy.

[51] J. L. TU - "The Baum-Connes conjecture and discrete group actions on trees", K-theory 17 (1999), p. 303-318.

[52] A. VAlette - "Introduction to the Baum-Connes conjecture", in Lectures in Mathematics, ETH, Zürich (1999), Birkhäuser, 2002. 